OPEN ACCESS

Edited by: Hugues Abriel,

University of Bern, Switzerland

Reviewed by:

Lane Brown,

Washington State University,

United States

Michael Craig Sanguinetti,

The University of Utah, United States

*Correspondence:

Boris Martinac

b.martinac@victorchang.edu.au

Charles D. Cox

c.cox@victorchang.edu.au

Specialty section:

This article was submitted to Pharmacology of lon Channels and

Channelopathies,

a section of the journal

Frontiers in Pharmacology

Received: 29 August 2021

Accepted: 03 November 2021

Published: 19 November 2021

Citation:

Zhou Z, Li JV, Martinac B and Cox CD

(2021) Loss-of-Function Piezo1

Mutations Display Altered Stability

Driven by Ubiquitination and

Proteasomal Degradation.

Front. Pharmacol. 12:766416.

doi: $10.3389 /$ fphar.2021.766416

\section{Loss-of-Function Piezo1 Mutations Display Altered Stability Driven by Ubiquitination and Proteasomal Degradation}

\author{
Zijing Zhou ${ }^{1}$, Jinyuan Vero $\mathrm{Li}^{1}$, Boris Martinac ${ }^{1,2 *}$ and Charles D. Cox ${ }^{1,2 *}$ \\ ${ }^{1}$ Molecular Cardiology and Biophysics Division, Victor Chang Cardiac Research Institute, Sydney, NSW, Australia, ${ }^{2}$ St. Vincent's \\ Clinical School, Faculty of Medicine, University of New South Wales, Sydney, NSW, Australia
}

Missense mutations in the gene that encodes for the mechanically-gated ion channel Piezo1 have been linked to a number of diseases. Gain-of-function variants are linked to a hereditary anaemia and loss-of-function variants have been linked to generalized lymphatic dysplasia and bicuspid aortic valve. Two previously characterized mutations, S217L and G2029R, both exhibit reduced plasma membrane trafficking. Here we show that both mutations also display reduced stability and higher turnover rates than wild-type Piezo1 channels. This occurs through increased ubiquitination and subsequent proteasomal degradation. Congruent with this, proteasome inhibition using $N$-acetyl-I-leucyl-I-leucyl-Inorleucinal (ALLN) reduced the degradation of both mutant proteins. While ALLN treatment could not rescue the function of $\mathrm{S} 217 \mathrm{~L}$ we show via multiple complementary methodologies that proteasome inhibition via ALLN treatment can not only prevent G2029R turnover but increase the membrane localized pool of this variant and the functional Piezo1 mechanosensitive currents. This data in combination with a precision medicine approach provides a new potential therapeutic avenue for the treatment of Piezo1 mediated channelopathies.

Keywords: post-translational modification (PMT), protein biosynthesis, mechanosensation, ubiquitiantion, proteasomal degradation

\section{INTRODUCTION}

Mechanosensitive (MS) ion channels are a structurally diverse class of cellular sensors that decode mechanical cues (Martinac and Cox, 2017; Cox et al., 2019; Poole, 2021; Syeda, 2021). The mechanosensitive channel Piezo1 has an emerging role in cardiovascular biology (Li et al., 2014; Rode et al., 2017; Douguet et al., 2019; Jiang et al., 2021; Yu et al., 2021) and is central in force sensing by vascular endothelial cells (Li et al., 2014; Ranade et al., 2014; Rode et al., 2017; Albarrán-Juárez et al., 2018; Nonomura et al., 2018). This channel responds to both membrane tension (Lewis and Grandl, 2015; Cox et al., 2016; Syeda et al., 2016) and shear stress (Ranade et al., 2014; Albarrán-Juárez et al., 2018; Maneshi et al., 2018; Lai et al., 2021) (whether these are separate molecular mechanisms remains to be determined) and is important in the development of valves in different tissues including the lymphatic system (Nonomura et al., 2018) and heart (Duchemin et al., 2019; Faucherre et al., 2020). Since its discovery and cloning (Coste et al., 2010) missense variants in the PIEZO1 gene have been linked to several human pathologies (Zarychanski et al., 2012; Albuisson et al., 2013; Bae et al., 2013; Lukacs et al., 2015; 
Glogowska et al., 2017; Murthy et al., 2017; Faucherre et al., 2020). In particular, loss-of-function missense variants in PIEZO1 have been linked to generalized lymphatic dysplasia (Fotiou et al., 2015; Lukacs et al., 2015) and bicuspid aortic valve (Faucherre et al., 2020).

Like many other integral membrane proteins, Piezol channels undergo biosynthetic quality control in the endoplasmic reticulum (ER) (Tannous et al., 2015). During this process Piezo1 undergoes significant $\mathrm{N}$-linked glycosylation, the process by which oligosaccharides are covalently attached to asparagine residues, which fulfils a critical role in its biosynthetic quality control (Li et al., 2020). These N-glycans may act as molecular tethers and participate in force sensing (particularly shear stress sensing) as shown for the epithelial sodium channel ENaC (Knoepp et al., 2020). Previously, we showed that trafficking defective Piezol mutations (disease linked or from structure function studies (Vero $\mathrm{Li}$ et al., 2021)) display modified N-linked glycosylation ( $\mathrm{Li}$ et al., 2021). Specifically, we noted that loss-of-function variants of Piezol such as S217L and G2029R show reduced N-linked glycosylation. Using the extensive published information from the $\mathrm{K}_{\mathrm{v} 11.1}$ channel literature we attempted through low temperature and pharmacological means (Robertson and January 2006; Smith et al., 2013; Anderson et al., 2014) to rescue surface expression and hence function of Piezol mutants but neither approach was successful.

As the first step of post-translational quality control, misfolded proteins are recognised and sent to the proteasome for degradation by a process termed ER-associated degradation or ERAD. ERAD is mostly, if not fully, dependent on the ubiquitin-proteasome system (UPS) (Ron and Walter, 2007; Christianson and Ye, 2014). The UPS represents the major quality-control machinery for guaranteeing the normal function of many proteins via eliminating misfolded protein products. Furthermore, shutting down the UPS using proteasome inhibitors can help trafficking-deficient mutants to escape ERAD and rescue the function of proteins such as; Msh2 (Arlow et al., 2013), RESA1 (Mohanraj et al., 2019) or $\mathrm{Na}_{\mathrm{v}} 1.1$ (Rusconi et al., 2007), both in vitro and in vivo.

In the current study, we confirmed that the disease-causing mutants S217L and G2029R of PIEZO1 are both traffickingdeficient. Moreover, we showed that both mutants exhibited ER retention, reduced protein stability and higher levels of ubiquitination, all of which are the hallmarks of ERAD. Inhibiting proteasomal degradation using ALLN reduced turnover of the mutant proteins. Importantly, the protein expression of G2029R was also stabilized using the clinically approved proteasome inhibitor Bortezomib (Chen et al., 2011). Finally, we showed that proteasome inhibition rescued the membrane targeting and increased stretchactivated currents in cells expressing G2029R. This raises the possibility that clinically available proteasome inhibitors could be used in conjunction with a precision medicine approach to treat a subset of loss-of-function PIEZO1 mutations.

\section{MATERIALS AND METHODS}

\section{Antibodies}

The following commercially available antibodies were used: antiGFP antibody (Santa Cruz Biotechnology, Dallas, TX, United States; 1:5,000 dilution); mouse monoclonal anti-Piezo1 antibody (Cat\# NBP2-88938, Novus Biologicals, Centennial, CO, United States; 1:1,000 dilution); mouse anti- $\alpha$-actinin antibody (Santa Cruz Biotechnology; 1:5,000 dilution); mouse anti- $\mathrm{Na}^{+} / \mathrm{K}^{+}$ ATPase antibody (a6F, DSHB; 1:1,000 dilution); mouse antiubiquitin antibody (clone FK2, ENZO, 1:1,000); rabbit antiGAPDH antibody (CellSignaling, 1:1,000); anti-rabbit IRDye680 (Li-Cor, 1:20,000); anti-mouse IRDye800 (Li-Cor, 1: 20,000); mouse anti-HA antibody (Sigma, 1: 200 for IF); Alexa Fluor 555 anti-mouse (ThermoFisher, 1:200 for IF). Unless specified, all the antibody dilutions refer to western blotting.

\section{Cell Culture and Transfection}

Piezo1 $^{-/-}$HEK293T cells (Lukacs et al., 2015) were a gift from Dr. Ardem Patapoutian (The Scripps Research Institute, La Jolla, CA, United States); HeLa cells were purchased from ATCC (Cell lines were not authenticated and were not listed in the database of commonly misidentified cell lines maintained by ICLAC (http:// iclac.org) and NCBI Biosample (http://www.ncbi.nlm.nih.gov/ biosample). All cell lines were confirmed to be mycoplasma free. HEK cells were transfected with polyethylenimine (PEI); HeLa cells were transfected with Lipofectamine 3,000 transfection reagent (ThermoFisher Scientific).

\section{Ubiquitination Assay}

Ubiquitination assay protocol is modified from previous work (Zhang et al., 2017; Cao et al., 2021). WT or mutant Piezo1 transfected cells were lysed in the co-IP buffer ( $1 \% \mathrm{w} / \mathrm{v}$ CHAPS, $0.6 \% \mathrm{w} / \mathrm{v}$ soy PC, $140 \mathrm{mM} \mathrm{NaCl}, 1 \mathrm{mM}$ EDTA, $25 \mathrm{mM} \mathrm{NaPIPES}$ ) supplemented with $2 \mathrm{mM}$ 1,4-Dithiothreitol (Sigma), 1× protease-inhibitor cocktail (Roche) and $20 \mathrm{mM} \mathrm{N}$ ethylmaleimide (E3876; Sigma), and the lysates were centrifuged at $16,000 \times \mathrm{g}$ for $10 \mathrm{~min}$ at $4^{\circ} \mathrm{C}$; the expression of each protein of interest was confirmed by immunoblotting $5 \%$ of the collected supernatants, and the remaining supernatants were incubated with $0.6 \mu \mathrm{g}$ of anti-GFP antibody and $10 \mu \mathrm{L}$ of Protein $\mathrm{G}$ Dynabeads (ThermoFisher) at $4^{\circ} \mathrm{C}$ overnight. The recovered beads were washed three times with the co-IP wash buffer (25 mM NaPIPES, $140 \mathrm{mM} \mathrm{NaCl}, 0.6 \%$ w/v CHAPS, $0.14 \%$ PC) supplemented with $2 \mathrm{mM}$ 1,4-Dithiothreitol (Sigma), 1× protease-inhibitor cocktail (Roche) and $20 \mathrm{mM}$ N-ethylmaleimide (E3876; Sigma), and heated in 2x sodium dodecyl sulphate (SDS)-PAGE loading buffer containing $1 \mathrm{M}$ urea and $10 \mathrm{mM}$ tris(2-carboxyethyl)phosphine (TCEP) at $62^{\circ} \mathrm{C}$ for $5 \mathrm{~min}$, and the eluted proteins were immunoblotted with anti-GFP or anti-ubiquitin antibodies.

\section{Live Cell Labelling}

For live cell membrane protein labelling, Piezo1 ${ }^{-1-}$ HEK293T cells were plated on 96 well clear bottom plate (ThermoFisher) coated with $0.1 \mathrm{mg} / \mathrm{ml}$ of Poly-L-Lysine 
(Sigma). Cells were transfected with pIRES-GFP constructs carrying WT or mutant Piezo1 (125 ng cDNA per well) with PEI. Sixty to $72 \mathrm{~h}$ after transfection, live labelling was performed by incubating the cells with anti-HA (Sigma, 1:100) antibody for $20 \mathrm{~min}$ at $37^{\circ} \mathrm{C}$. The cells were then washed with DMEM six times before being incubated with Alexa Fluor 555 anti-mouse secondary antibody (1:200) for $15 \mathrm{~min}$ at room temperature $\left(22^{\circ} \mathrm{C}\right)$. Cells were washed again 5 times with DMEM and twice with phosphate buffered saline (PBS), then fixed with $4 \%$ paraformaldehyde (PFA) for $20 \mathrm{~min}$ at room temperature. PFA was washed off and replaced with PBS before confocal analysis.

For co-labelling of Piezol and the endoplasmic reticulum (ER), HeLa cells were transfected with WT or mutant Piezol fused with GFP. After transfection for 60-72 h, cells were washed with PBS twice then incubated with $1 \mu \mathrm{M}$ of ER-Tracker Red dye (Invitrogen) for $20 \mathrm{~min}$ at $37^{\circ} \mathrm{C}$. The cells were washed again with PBS three times and fixed with 4\% PFA for $20 \mathrm{~min}$ at room temperature. PFA was then replaced with PBS, and the ER or Piezo1-GFP signals were visualized using confocal microscopy (Zeiss LSM 700 inverted).

\section{Proteasomal and Lysosomal Inhibitor Treatment}

Piezo1 $^{-/-}$HEK293T cells were transfected with GFP fused WT or mutant Piezol before treatment. The transfected cells were treated with the following conditions: $10 \mu \mathrm{g} / \mathrm{ml}$ of $\mathrm{CHX}$ (Sigma) for 4 or $8 \mathrm{~h} ; 10 \mathrm{mM}$ of $\mathrm{NH}_{4} \mathrm{Cl}$ (Sigma) or $10 \mu \mathrm{M}$ of ALLN (Sigma) for 9 or $24 \mathrm{~h} ; 1$ or $10 \mathrm{nM}$ of Bortezomib (BTZ) for $24 \mathrm{~h}$. The cells were then lysed in radioimmunoprecipitation assay (RIPA) buffer ( $150 \mathrm{mM} \mathrm{NaCl}, 1.0 \% \mathrm{w} / \mathrm{v} \mathrm{NP} 40,0.5 \% \mathrm{w} / \mathrm{v}$ sodium deoxycholate, $0.1 \% \mathrm{w} / \mathrm{v}$ SDS, $50 \mathrm{mM}$ Tris, $\mathrm{pH} 7.4$ ) supplemented with $1 \times$ protease-inhibitor cocktail and $10 \mathrm{mM}$ tris(2-carboxyethyl)phosphine (TCEP). Cell lysates were subjected to western blotting.

\section{Electrophysiology}

Transiently transfected Piezo1 ${ }^{-/-}$HEK293T cells were plated on $35 \mathrm{~mm}$ dishes for patch clamp analysis. The extracellular solution for cell-attached patches contained high $\mathrm{K}^{+}$to zero the membrane potential; it consisted of $90 \mathrm{mM}$ potassium aspartate, $50 \mathrm{mM} \mathrm{KCl}$, $1 \mathrm{mM} \mathrm{MgCl} 2$ and $10 \mathrm{mM}$ HEPES (pH 7.2) adjusted with $5 \mathrm{M}$ $\mathrm{KOH}$. The pipette solution contained $140 \mathrm{mM} \mathrm{CsCl}$ with $10 \mathrm{mM}$ HEPES (pH 7.2) adjusted with the respective hydroxide. Ethylene glycol-bis( $\beta$-aminoethyl etlier)-N,N, $\mathrm{N}^{\prime}, \mathrm{N}^{\prime}$-tetraacetic acid (EGTA) was added to control levels of free pipette (extracellular) $\mathrm{Ca}^{2+}$ using the online EGTA calculator-CaEGTA Calculator TS v1.3-Maxchelator. Negative pressure was applied to patch pipettes using a High-Speed Pressure Clamp-1 (ALA Scientific Instruments, Farmingdale, NY, United States) and recorded in millimetres of mercury (mmHg) using a piezoelectric pressure transducer (WPI, Sarasota, FL, United States). Borosilicate glass pipettes (SigmaAldrich) were pulled with a vertical pipette puller (PP-83, Narashige, Tokyo, Japan) to produce electrodes with a resistance of 1.8-2.2 M $\Omega$. The Piezol currents were amplified using an AxoPatch 200B amplifier (Axon Instruments, Union City, CA, United States), and data were sampled at a rate of $10 \mathrm{kHz}$ with $1 \mathrm{kHz}$ filtration and analysed using pCLAMP10 software (Axon Instruments).

\section{Western Blotting}

Transiently transfected cells were solubilized in a modified RIPA buffer [Tris buffer $10 \mathrm{mM}$, ethylenediaminetetraacetic acid (EDTA) $1 \mathrm{mM}, \quad \mathrm{NaCl} 140 \mathrm{mM}$, in $(\% \mathrm{w} / \mathrm{v})$ : Sodium deoxycholate 0.1 , SDS 0.1 , Triton X-100 1.0, $\mathrm{pH}$ 7.2] supplemented with $1 \times$ EDTA-free protease inhibitor cocktail tablets (Sigma-Aldrich), $1 \mathrm{mM}$ (phenylmethylsulfonyl fluoride) PMSF, $10 \mathrm{mM}$ TCEP, and $1 \mathrm{mM} \mathrm{N}$-ethylmaleimide (NEM) for 10 min on a rotating wheel at $4^{\circ} \mathrm{C}$. Cell lysates were cleared by centrifugation at $13,000 \times \mathrm{g}$ at $4^{\circ} \mathrm{C}$ for $10 \mathrm{~min}$. For quantitative western blot analysis, GFP-fused Piezol was probed with a rabbit monoclonal anti-GFP antibody (Santa Cruz Biotechnology, Dallas, TX, United States); or a mouse monoclonal anti-Piezo1 antibody (Cat\# NBP2-88938, Novus Biologicals, Centennial, CO, United States); mouse anti- $\alpha$-actinin antibody (Santa Cruz Biotechnology) was added simultaneously for a loading comparison followed by anti-rabbit IRDye680 and anti-mouse IRDye800 (Li-Cor) to enable quantification with the LI-COR Odyssey system (LI-COR Biotechnology, Lincoln, NE, United States).

\section{Biotinylation}

For each well in a 6-well plate, transiently transfected cells were washed three times using ice cold PBS and then incubated with biotin buffer (154 mM NaCl, $10 \mathrm{mM}$ HEPES, $3 \mathrm{mM} \mathrm{KCL}, 1 \mathrm{mM}$ $\mathrm{MgCl}_{2}, 0.1 \mathrm{mM} \mathrm{CaCl}_{2}, 10 \mathrm{mM}$ glucose, $\mathrm{pH}$ 7.6) containing $1 \mathrm{mg} /$ $\mathrm{ml}$ Sulfo-NHS-Biotin (ThermoFisher Scientific, EZ-Link ${ }^{\mathrm{TM}}$, Lot. No. TI266926) for $1 \mathrm{~h}$ on ice. The biotin buffer was then washed off and quenched using $50 \mathrm{mM}$ of Glycine in PBS for $10 \mathrm{~min}$ on ice. Cells were then washed three times using ice cold PBS and solubilized using $250 \mu \mathrm{L}$ RIPA buffer. Cell lysates were cleared by centrifugation at $13,000 \times \mathrm{g}$ at $4^{\circ} \mathrm{C}$ for $10 \mathrm{~min} 20 \mu \mathrm{L}$ supernatant was taken and supplemented with $2 \% \mathrm{w} / \mathrm{v}$ SDS and $0.8 \mathrm{M}$ urea and designated as the "input" sample. The remaining supernatant was incubated overnight at $4^{\circ} \mathrm{C}$ with $20 \mu \mathrm{L}$ Streptavidin-Agarose beads (Sigma-Aldrich, Lot\#: SLBR5741V) blocked using 0.5\% w/v bovine serum albumin for $1 \mathrm{~h}$. Beads were collected and washed three times using RIPA buffer. For protein elution, beads were loaded with $20 \mu \mathrm{L} 0.9 \%$ w/v NaCl solution supplemented with $2 \%$ SDS and $0.8 \mathrm{M}$ urea, and heated at $62^{\circ} \mathrm{C}$ for $5 \mathrm{~min}$. Then they were centrifuged at $500 \times \mathrm{g}$ at $4^{\circ} \mathrm{C}$ for $1 \mathrm{~min}$ in the column, and the biotinylated eluate was collected. Identical protein amounts from input and biotinylated samples were loaded for western blotting then probed with; mouse monoclonal anti-Piezol antibody (Cat. No. NBP2-75617, Novus Biologicals), rabbit anti-GAPDH (CellSignaling) and mouse anti- $\mathrm{Na}^{+} / \mathrm{K}^{+}$ATPase antibody $(\mathrm{a} 6 \mathrm{~F}$, DSHB).

\section{Statistics}

Protein bands in western blots were quantified using ImageJ software, and peak currents were measured using Clampfit software. All data are expressed as means \pm SEM; $n$ represents 
A

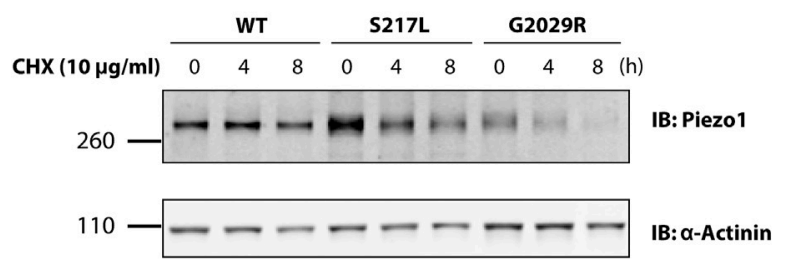

C

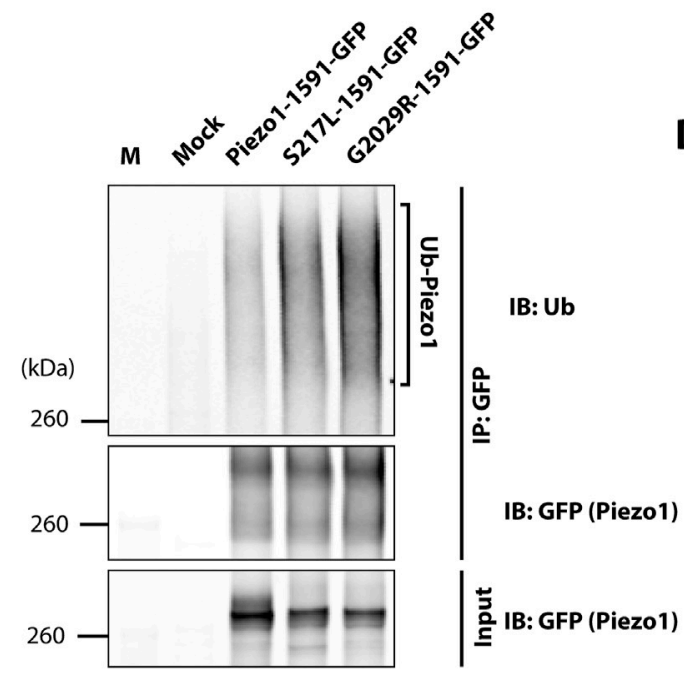

B

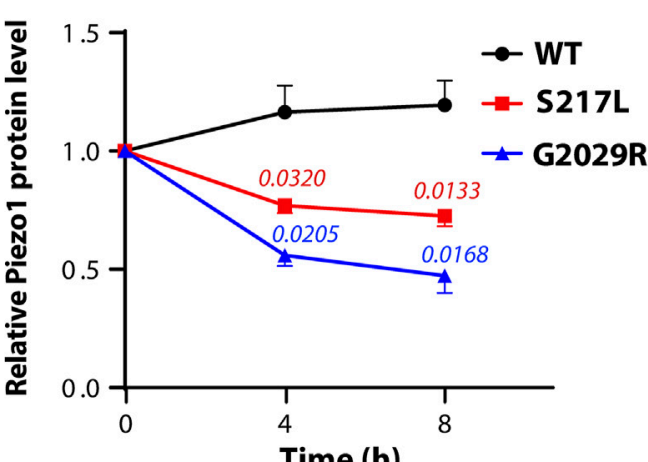

Time (h)

D

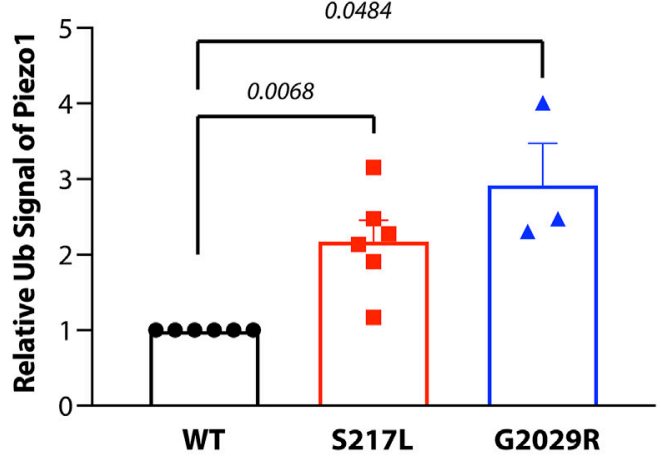

FIGURE 1 | Instability of Piezo1 loss-of-function mutants correlates with increased ubiquitination. (A) Piezo1 $1^{-/}$HEK293T cells overexpressing wild-type (WT) or mutant Piezo1-GFP were treated with $10 \mu \mathrm{g} / \mathrm{ml}$ cycloheximide (CHX) for 0,4 or $8 \mathrm{~h}$ before being collected for western blotting. (B) Summary data of panel (A) showing Piezo1 protein levels normalized to $\alpha$-Actinin. ( ${ }^{*} p=0.0320$ and ${ }^{*} p=0.0133$ for S217L at 4 and 8 h; ${ }^{*} p=0.0180$ and ${ }^{*} p=0.0168$ for G2029R at 4 and 8 h) (C) WT or mutant Piezo1-GFP expressed in Piezo $1^{-/-}$HEK293T cells were immunoprecipitated using an anti-GFP antibody and the eluted fraction and input cell lysate were probed for Piezo1 levels. An immunoblot of the immunoprecipitated protein using a mono- and poly-ubiquitin (Ub) antibody is also shown (M = marker). (D) Summary data of panel $\mathrm{C}$ showing the level of Piezo1 ubiquitination normalized to the level of immunoprecipitated Piezo1 protein ( $n=4$ for all experiments). One way ANOVA with Dunnett's multiple comparison test was used for statistical analysis.

the number of independent biological replicates. $p<0.05$ was considered statistically significant.

\section{RESULTS}

\section{S217L and G2029R Piezo1 Are Less Stable Compared to Wild-type Piezo1}

S217L and G2029R Piezol are both loss-of-function mutants (Faucherre et al., 2020; Lukacs et al., 2015; Li et al., 2020). The exact mechanisms that underlie this loss-of-function have not been fully explored. The cycloheximide chase procedure has permitted visualization of the degradation kinetics of the steady state population of a variety of cellular proteins (Kao et al., 2015). To understand the turnover rate of Piezol channels, we applied cycloheximide (CHX) to the culture media of $\mathrm{Piezol}^{-/-}$ HEK293T cells expressing wild-type (WT) or mutant Piezo1 (S217L and G2029R) fused to GFP (Cox et al., 2016; Li et al., 2020; Vero Li et al., 2021; Ridone et al., 2020; Buyan et al., 2020).
The protein level of WT Piezol was stable and did not decrease even after $8 \mathrm{~h}$ treatment with $10 \mu \mathrm{g} / \mathrm{ml}$ of CHX. In comparison, both S217L and G2029R showed significantly reduced protein amounts after 4- or 8-h treatment compared to untreated controls. Specifically, we observed a 27 or $53 \%$ reduction in protein level for S217L and G2029R, respectively, after 8-h treatment, implying a faster turnover rate of the mutant proteins (Figures 1A,B). One potential mechanism that could drive faster protein turnover is ubiquitination. Protein ubiquitination is one of the key processes in endoplasmic reticulum associated degradation (ERAD) enabling the elimination of mis-folded proteins (Ron and Walter, 2007; Christianson and Ye, 2014). As a result, we examined the ubiquitination levels of Piezol and mutant Piezol proteins expressed in Piezol $^{-1-}$ HEK293T cells. To do this, we immunoprecipitated overexpressed Piezo1 and analysed the ubiquitination signal specific to Piezo1 in the elution (Figure 1C). The normalized ubiquitination levels of both S217L and G2029R were 2.2 and 2.8-fold higher respectively than WT. Interestingly, the GLD linked mutant protein G2029R 

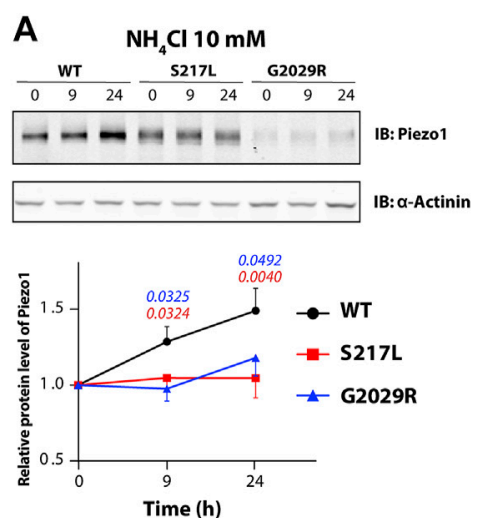

B
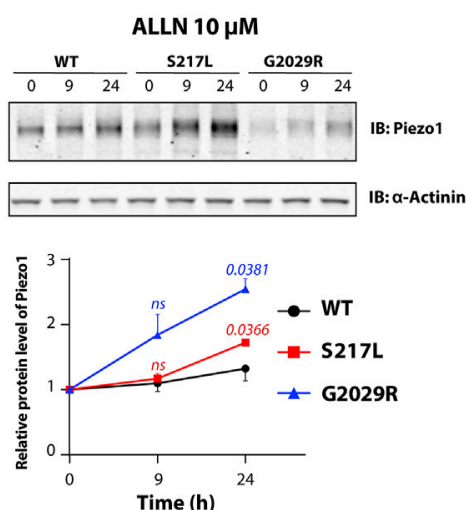
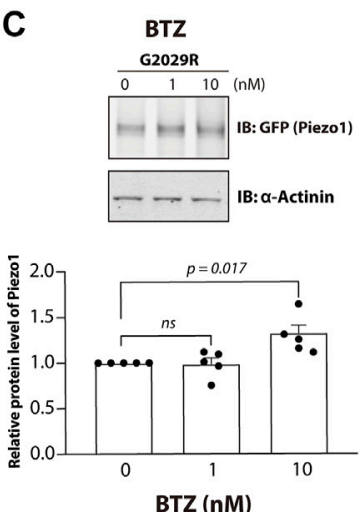

FIGURE 2 |S217L and G2029R Piezo1 mutants go through proteasomal degradation. Piezo1 ${ }^{-/}$HEK293T cells overexpressing WT or mutant Piezo1 were treated with (A) a lysosomal degradation inhibitor $\mathrm{NH}_{4} \mathrm{Cl}$ or (B) a proteasomal degradation inhibitor ALLN for 0, 9 or $24 \mathrm{~h}$. Representative results are shown in the upper panel. Lower panel, summary data of the corresponding experiments with comparison between mutant and WT Piezo1 shown in blue for G2029R and in red for S217L. (C) Piezo1 $^{-/}$HEK293T cells overexpressing G2029R Piezo1 were treated with 0, 1 or $10 \mathrm{nM}$ of Bortezomib for $24 \mathrm{~h}$ before being collected for western blot. Upper panel, representative western blot. Lower panel, summary data of the western blot experiments. One way ANOVA with Dunnett's multiple comparison test was used for statistical analysis (ns = not significant).

seemed to have a higher ubiquitination level than that of S217L (Figures 1C,D).

\section{Degradation of S217L and G2029R Piezo1 Is Proteasome Dependent}

Proteasome dependent degradation, in addition to ubiquitination, is another hallmark of ERAD. To investigate the degradation pathway for WT and mutant Piezo1, we utilized $\mathrm{NH}_{4} \mathrm{Cl}$ a generic inhibitor of lysosomal degradation and a non-specific inhibitor of proteasomal degradation acetyll-leucyl-l-leucyl-l-norleucinal (ALLN). Treatment with $10 \mathrm{mM}$ of $\mathrm{NH}_{4} \mathrm{Cl}$ caused an increase in the levels of WT Piezol protein. Relative WT Piezol protein levels were increased by $49 \%$ after treatment for $24 \mathrm{~h}$. This is consistent with WT Piezol being degraded by a lysosomal pathway. In contrast $\mathrm{NH}_{4} \mathrm{Cl}$ had little effect on the protein levels of S217L and G2029R (Figure 2A). In contrast to that, both S217L and G2029R showed significantly increased protein expression (72 and 155\% increase in protein level respectively for 24 -h treatment) in response to ALLN treatment (Figure 2B). Importantly, the level of G2029R protein expression increased more than that of S217L, which was consistent with the higher level of G2029R ubiquitination and faster turnover rate determined using CHX treatment (Figures 1B,D). We further asked whether the ability of ALLN to reduce G2029R protein turnover could be recapitulated using a specific proteasome inhibitor. To test this, we used the clinically available anti-cancer agent Bortezomib (BTZ) and treated Piezo1 ${ }^{-/-}$ HEK293T which overexpressed the G2029R mutant with concentrations that ranged from $0.1-10 \mathrm{nM}$. The protein level of the G2029R mutant was significantly increased after $24 \mathrm{~h}$ treatment with BTZ at $10 \mathrm{nM}$ when compared to untreated controls (Figure 2C). Collectively our data indicates that both S217L and G2029R Piezo1 go through ER associated degradation when overexpressed in HEK293T cells.

\section{Aberrant Membrane Targeting of S217L and G2029R Piezo1}

Mis-localization of G2029R has been previously reported (Lukacs et al., 2015) and replicated (Li et al., 2020). However, conflicting reports exist regarding the sub-cellular localization of S217L (Faucherre et al., 2020; Li et al., 2020). To investigate the membrane targeting efficiency of these two mutants, we performed membrane biotinylation by biotinylating the membrane proteins and pulling down those proteins with streptavidin conjugated agarose beads. The Piezo1 signal in the membrane fraction for the two mutants, normalized to $\mathrm{Na}^{+} / \mathrm{K}^{+}$-ATPase and input Piezo1, was significantly reduced compared to WT Piezo1 by $>50 \%$ (Figures 3A,B), suggesting aberrant trafficking and inefficient membrane targeting of both S217L and G2029R. To further corroborate these observations, we studied the localization of WT and mutant Piezo1 by overexpressing GFP fused Piezo1 in HeLa cells, and then imaged live cells stained with a marker for the ER using confocal microscopy. Unlike the WT Piezo1, which was largely localized to the plasma membrane, S217L and G2029R Piezo1 were both largely confined to the ER indicated by the high level of co-localization with the signal from the ER tracker (Figure 3C). We quantified the degree of co-localization between the Piezol-GFP fusion proteins and the ER tracker and observed that the co-localization co-efficient for WT Piezol with the ER tracker was $>1.8$ fold less than that for the mutant proteins (Figure 3D).

\section{ALLN Is Able to Rescue Membrane Localization of G2029R Piezo1}

Rescuing the membrane localization and/or function of ion channel mutations, which are degraded by ERAD, with proteasomal degradation inhibitors or by modifying levels 
A

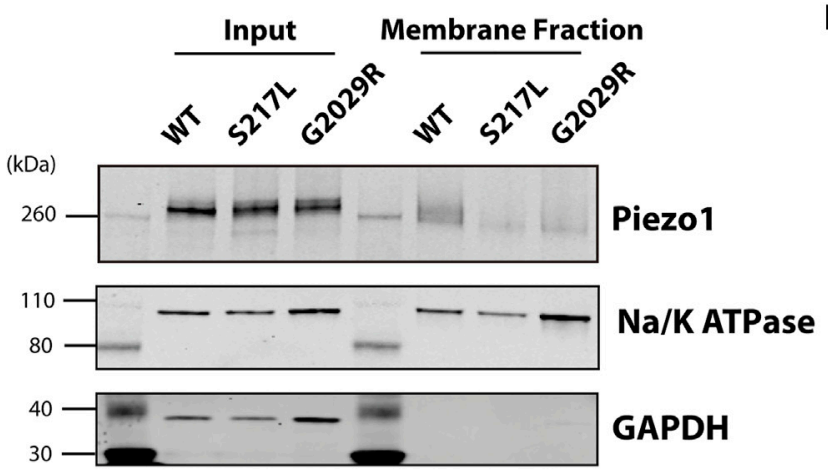

C
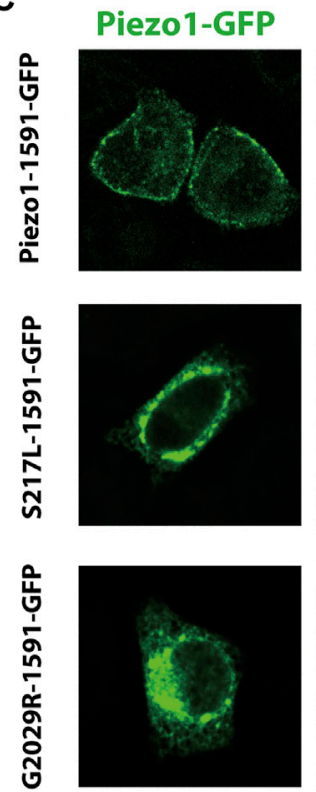

B
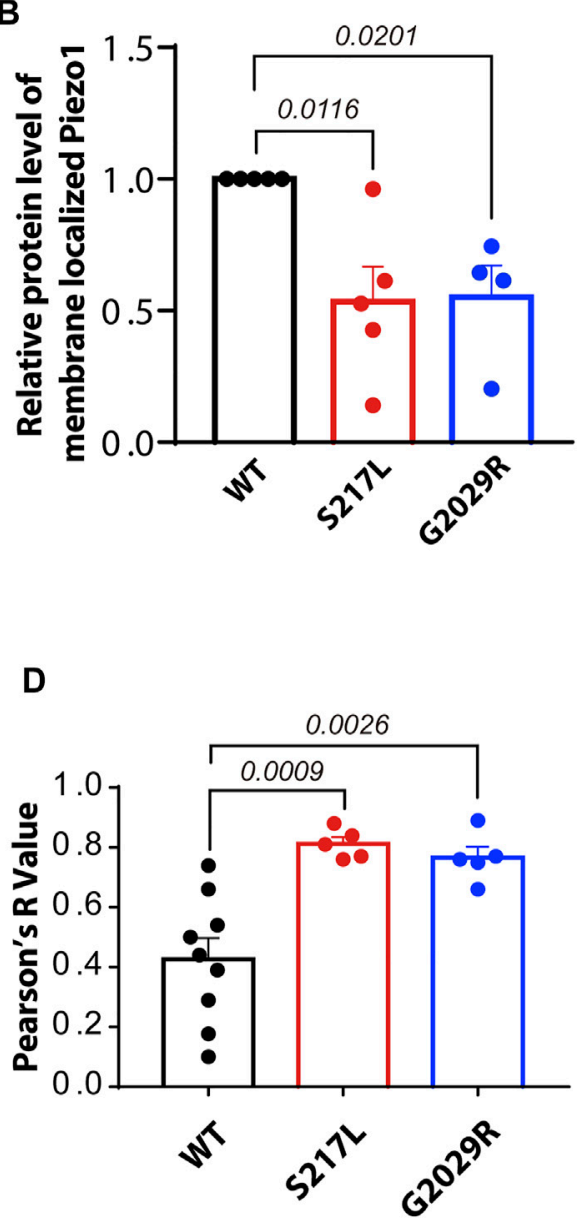

FIGURE 3 | S217L and G2029R are trafficking-deficient mutants. (A) Western blot showing the membrane localized fraction of WT and mutant Piezo1 proteins Piezo1 from Piezo1 ${ }^{-1-}$ HEK293T cells using a standard biotinylation assay. The membrane fraction of the eluted lysate is visualised using the ubiquitous membrane protein $\mathrm{Na}^{+} / \mathrm{K}^{+}$ATPase and the cytosolic protein GAPDH is shown as a comparison for whole protein lysate (Input) and bead elution (Membrane Fraction). (B) Summary data of panel (A). One way ANOVA with Dunnett's multiple comparison test was used for statistical analysis. (C) HeLa cells overexpressing WT or mutant Piezo1 with GFP tag were stained with ER tracker and analysed using confocal microscopy. Notice that the WT Piezo1 is localized to the plasma membrane while the mutant Piezo1 co-localizes almost exclusively with the ER tracker. Scale bar, $10 \mu \mathrm{m}$. (D) Summary data showing the overlap of GFP (Piezo1) and the ER signal indicated by Pearson's R value. One way ANOVA with Dunnett's multiple comparison test was used for statistical analysis.

of protein ubiquitination have been demonstrated by several independent studies (Rusconi et al., 2007; Arlow et al., 2013; Mohanraj et al., 2019; Kanner et al., 2020). Given that the protein turnover of both S217L and G2029R Piezol can be influenced by ALLN treatment, we asked if ALLN also affected the membrane localization of these two mutants. We conducted live cell labeling by overexpressing a Piezol construct that contains an extracellular HA tag (Piezo1897-HA) integrated into a pIRES2-EGFP vector. The HA tag was inserted within an extracellular loop previously shown to be amenable to the insertion of a Myc tag in a similar region in mouse Piezol (Coste et al., 2015). Indeed, we tested the function of human Piezo1-897-HA using cellattached patch clamping and the peak currents elicited in response to negative pressure from Piezo1-897-HA were comparable to those elicited by WT Piezol when they were overexpressed in Piezo1 ${ }^{-1-}$ HEK293T, indicating the function and localization of Piezo1-897-HA is similar to WT (Figure 4A). We then generated S217L and G2029R mutants based on the same construct by site-directed mutagenesis. Both mutants behaved differently from WT; the membrane labeling intensity was much lower than the wild-type Piezo1. Importantly, upon ALLN treatment, membrane intensity increased by more than 9 folds for G2029R but not for S217L Piezo1 (Figures 4B-F). Our data indicated that membrane localization of G2029R Piezol can be partially rescued by the proteasomal inhibitor ALLN. 
A

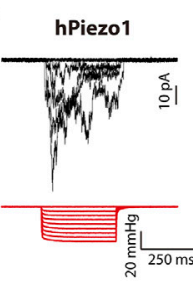

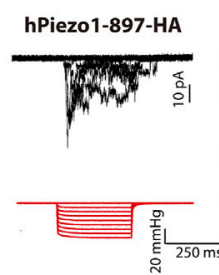

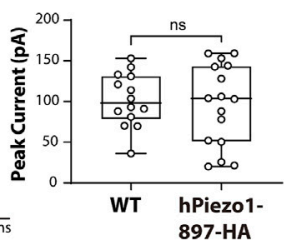

C
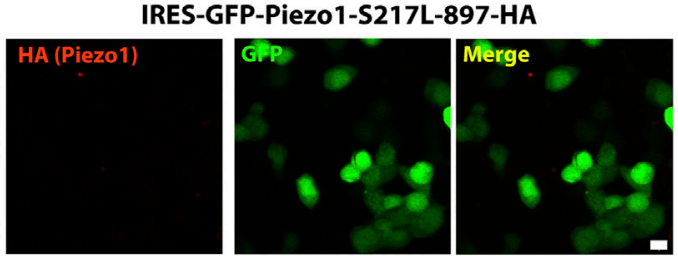

D
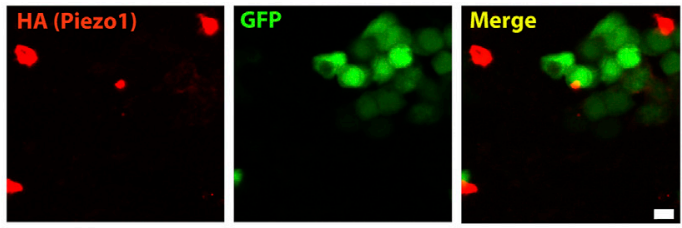

E

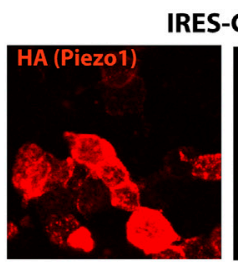

IRES-GFP-Piezo1-897-HA

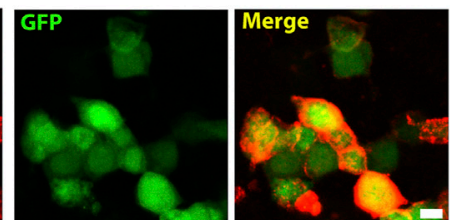

B
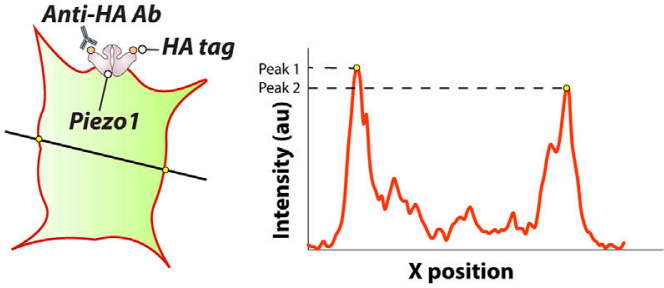

IRES-GFP-Piezo1-S217L-897-HA + ALLN $10 \mu \mathrm{M}$

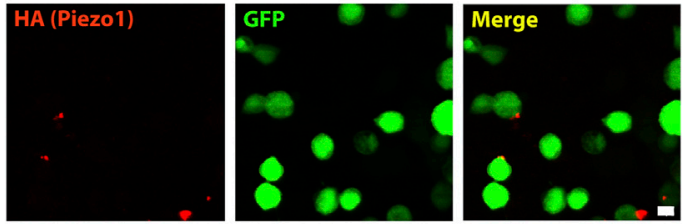

IRES-GFP-Piezo1-G2029R-897-HA + ALLN $10 \mu \mathrm{M}$
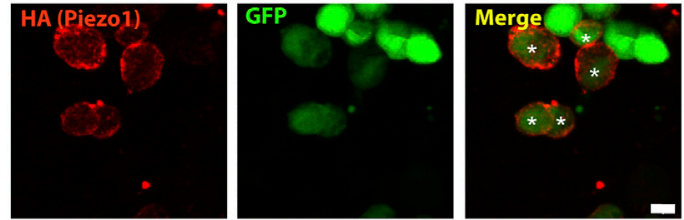

$\mathbf{F}$

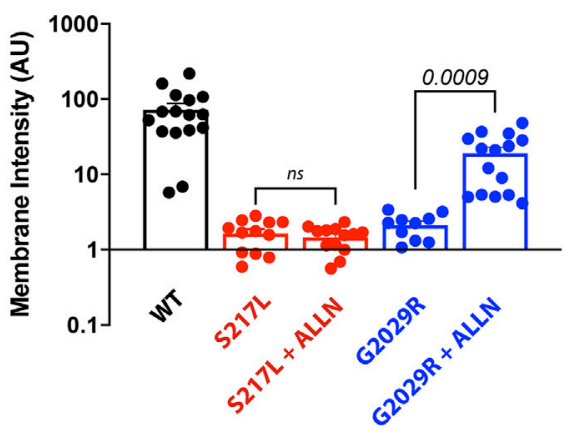

FIGURE 4 | ALLN partially rescues the membrane-localization of G2029R. (A) Piezo1 ${ }^{-/}$HEK293T cells overexpressing WT or Piezo1-897HA were subjected to cell-attached patch clamping. Representative traces in response to square wave negative pressure pulses are shown. The right panel shows the summary data of peak currents elicited from cell-attached patches as a box and whiskers plot illustrating the minimum and maximum. (B) Schematic showing how the membrane intensity is calculated. A straight line was drawn across the GFP positive cells with only the GFP channel visible to preclude bias. The peak value of the HA signal within the membrane region, determined by GFP channel and bright field, was determined using Image J's Plot Profile tool. Peak values on each side of the cell were averaged as one data point. (C-E) Piezo1 ${ }^{-1-}$ HEK293T cells overexpressing WT or mutant Piezo1 with an extracellular 897-HA tag were stained with anti-HA antibody and Alexa-555 goat anti mouse secondary antibody before PFA fixation. All figures were taken under the same optical configuration and presented with the same maximum/minimum intensity. Red channel, signal from HA antibody; green channel, signal from free GFP. Scale bar, $10 \mu m$. (F) Summary data from Panels (C-E). Y axis represents membrane intensity in arbitrary fluorescent units. Comparison between treated and untreated cells was caried out using Student's t-test.

\section{Effect of ALLN Treatment on Mechanically-Evoked Currents}

Given that ALLN increased the membrane targeting of G2029R Piezol we examined whether the increased membrane localized G2029R Piezo1 correlated with an increase in mechanically evoked currents. We therefore conducted cell-attached patch clamping with Piezo1 ${ }^{-/-}$HEK293T cells overexpressing the WT or mutant Piezo1. We first examined the mechanosensitive currents from WT Piezo1, with and without ALLN treatment. The peak currents displayed only minor differences when comparing between the two groups, suggesting ALLN did not influence WT Piezol channel activity (Figures 5A,B). We then studied the electrophysiological behavior of S217L and G2029R Piezo1 with and without ALLN treatment. Consistent with our live labeling data, both ALLN untreated or treated S217L Piezo1 did not respond to mechanical stimuli, while the peak currents of G2029R Piezo1 increased by more than 7.5-fold after ALLN treatment (Figures 5A,B). This data suggested ALLN was able to increase both the membrane localization and mechanically evoked currents from heterologously expressed G2029R 
A
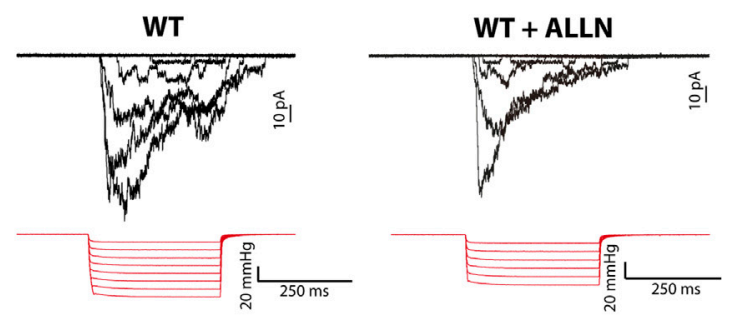

S217L
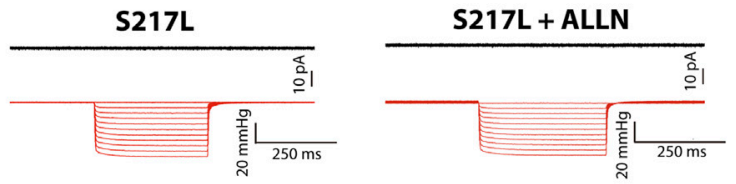

G2029R
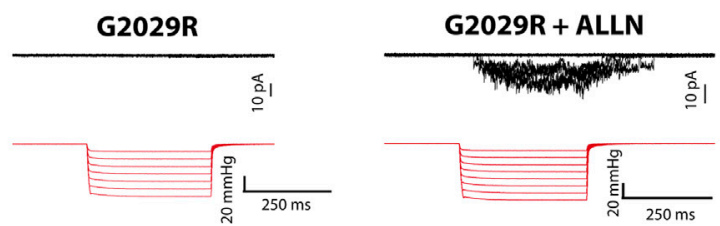

B

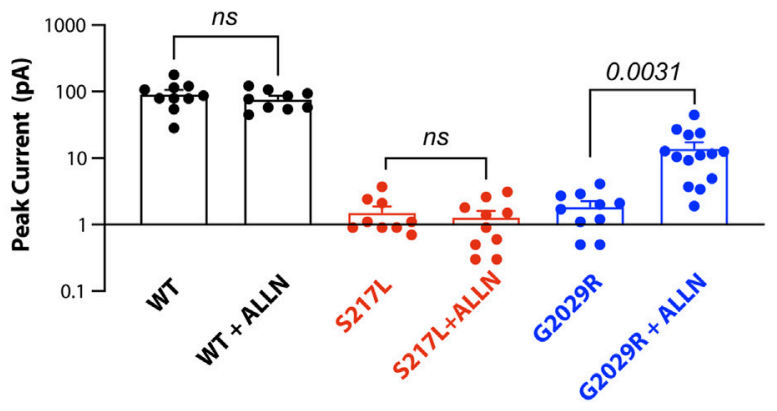

FIGURE 5 | Mechanically-evoked G2029R currents are increased by ALLN treatment. (A) Piezo1 $1^{-1-}$ HEK293T cells overexpressing WT or mutant Piezo1 were treated with or without ALLN for 10-15 h before cell-attached patch clamping. Representative traces are shown. (B) Summary data from panel (A) illustrating the peak currents elicited from cell-attached patches from Piezo1 ${ }^{-/-}$HEK293T cells overexpressing WT or mutant Piezo1 with or without ALLN treatment. $p$ values determined using Student's t-test to compare treated and untreated cells.

Piezo1. Furthermore, this compounds the idea that the mislocalization and increased turnover rate of G2029R Piezo1 serves as a major reason for its loss-of-function.

\section{DISCUSSION}

PIEZO1 variants have been linked to a number of pathologies (Zarychanski et al., 2012; Albuisson et al., 2013; Bae et al., 2013; Fotiou et al., 2015; Lukacs et al., 2015; Faucherre et al., 2020). The mutations S217L and G2029R are loss-of-function disease-linked PIEZO1 variants, identified by whole-exome sequencing (Lukacs et al., 2015; Faucherre et al., 2020). Specifically, S217L has been liked to bicuspid aortic valve while G2029R contributes to generalized lymphatic dysplasia. In combination with evidence that loss-of-function PIEZO1 variants are linked to varicose veins (Faucherre et al., 2020) it seems clear that Piezol has an emerging role in valvular formation and function in a number of settings (Nonomura et al., 2018; Faucherre et al., 2020). However, we are only beginning to understand the exact molecular mechanisms underlying the molecular dysfunction of these variants and other disease-linked PIEZO1 variants.

Loss-of-function variants of ion channels are likely to arise due to two broad mechanisms: 1) aberrant localization or 2) gating defects (potentially increased inactivation or reduced force sensitivity in the case of Piezol channels). Previous work points towards defective trafficking of G2029 R (Lukacs et al., 2015) but that S217L had normal membrane localization (Faucherre et al., 2020). We showed that both S217L and G2029R displayed reduced N-glycosylation (Li et al., 2020) [in addition to other trafficking defective mutants generated during structure-function studies (Vero Li et al., 2021)] similar to trafficking defective disease causing variants in ion channels such as $\mathrm{K}_{\mathrm{v} 11.1}$, which causes long QT syndrome type 2 (Vandenberg et al., 2012). Herein we provided further insight into the molecular mechanisms by which these mutations influence Piezol function.

First, we observed that both S217L and G2029R had increased protein turnover rates when compared to WT Piezol protein. Using a standard cycloheximide treatment strategy to inhibit new protein synthesis it was immediately clear that both mutants were less stable at the protein level. To begin to understand the mechanism of this reduced stability and increased turnover

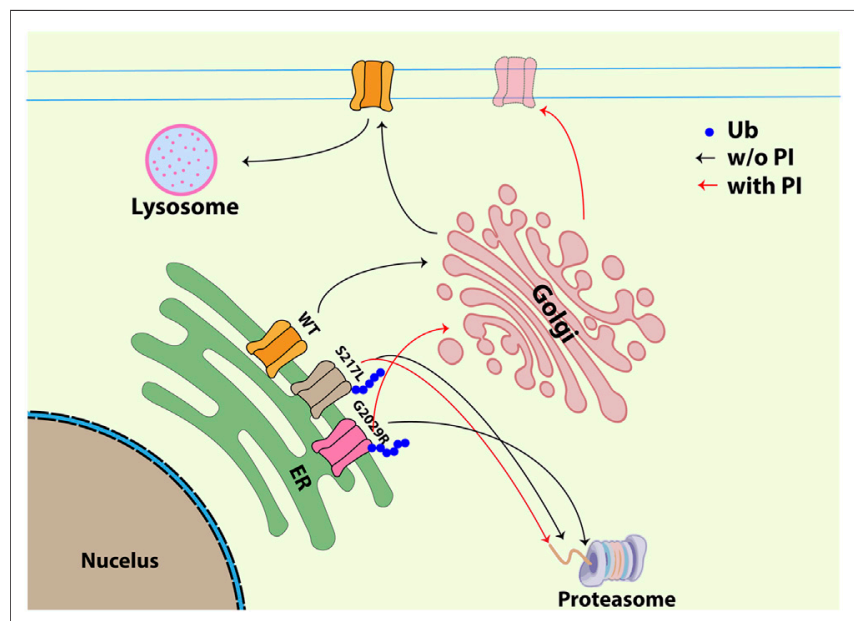

FIGURE 6 | Schematic figure illustrating the trafficking pathway for WT, S217L and G2029R with or without ALLN. WT Piezo1 transits from ER to plasma membrane and is finally degraded through membrane-protein internalization and lysosome dependent degradation. Unlike the WT Piezo1, both S217L and G2029R Piezo1 go through ubiquitination and proteasome dependent degradation. Treatment with the proteasomal inhibitor ALLN did not influence the membrane localization of S217L, while G2029R was partially rescued exhibiting increased membrane labelling and increased amplitudes of mechanically-evoked currents. Black arrow indicates the trafficking pathway for all Piezo1 proteins without ALLN treatment; red arrow indicates the trafficking pathway for S217L and G2029R Piezo1 after ALLN treatment (Ub-ubiquitin, P/l-proteasomal inhibition). 
rate, that we had identified previously in mutants with multiple N-linked glycosylation sites mutated (Li et al., 2020), we looked at Piezo1 ubiquitination. We showed that both loss-of-function mutations (S217L and G2029R) underwent increased ubiquitination. This suggested that the degradation of these mutants was likely driven by the proteasome. To test this hypothesis, we treated HEK293T cells expressing Piezo1 with the broad-spectrum proteasome inhibitor ALLN. Consistent with our ubiquitination data ALLN significantly increased the protein levels of both S217L and G2029R. We also showed that the clinically used, and more specific, proteasome inhibitor bortezomib (Chen et al., 2011) at nM concentrations could also reduce the turnover rate of G2029R and boost protein levels. In contrast it seemed the primary route for the degradation of WT Piezol in a heterologous expression system involved a lysosomal pathway that was inhibited by $\mathrm{NH}_{4} \mathrm{Cl}$.

The degradation of proteins through the proteasome is a common route for the disposal of misfolded proteins. Our imaging in HeLa cells showed that both S217L and G2029R exhibited significant retention in the ER consistent with a trafficking defect and our previous data (Li et al., 2020). Although at first glance the level of co-localization with ER tracker in HeLa cells seems not to quantitatively match the differences in current density measured using patch clamping it is important to note that co-localization of ER tracker and WT Piezol is expected as the WT protein needs to transit through ER prior to reach its destination at the plasma membrane. When we probed the membrane localized pool of these mutants using membrane biotinylation we did however see some membrane labelling of S217L and G2029R (consistent with the incomplete co-localization of ER tracker and Piezol mutants in HeLa cells) but this was significantly reduced compared to WT Piezo1. Some level of membrane labelling is consistent with the immunogold labelling we previously carried out to look at subcellular localization of these mutant proteins (Li et al., 2020). This G2029R data also fits with the small whole-cell indentation currents documented in the first paper implicating G2029R in disease (Lukacs et al., 2015). Altogether these data provide strong support that both S217L and G2029R are largely retained in the ER. The lack of normal trafficking and membrane localization of S217L and G2029R therefore likely provides a significant contribution to the loss-offunction phenotype of these two mutants. However, the residual amount of membrane localised S217L in particular when combined with the complete abolition of stretchactivated currents means we cannot completely rule out an additional gating phenotype in this variant.

Several strategies have been employed to rectify trafficking defects in ion channels that lead to channelopathies as a potential route to therapies. This includes a recent report that the reversal of ubiquitination may act as a potential broadspectrum strategy to rectify channel trafficking defects (Kanner et al., 2020). We previously used N-linked glycosylation as a surrogate of membrane trafficking and tried to use two widely used strategies, low temperature and a chaperone (Anderson et al., 2006; Robertson and
January 2006; Anderson et al., 2014), to improve the trafficking of G2029R. Neither strategy was successful (Li et al., 2020). In the current study given the robust effects of ALLN on mutant Piezo1 protein levels we next asked the question of whether proteasome inhibition could rescue trafficking of Piezol mutants as shown for other ion channels (Wang et al., 2018).

We showed using both a live labelling approach, similar to previous studies (Lukacs et al., 2015; Saotome et al., 2018), and patch clamp electrophysiology that ALLN not only could increase the membrane labelling of G2029R but could also cause a modest increase in the cell-attached mechanically-evoked currents (Figure 6). The S217L live cell labelling and stretch-activated currents were not affected by ALLN even though it did slow the rate of protein turnover. This is not surprising given that for $\mathrm{K}_{\mathrm{v} 11.1}$ there is a continuum where some channel mutations can be rescued by low temperature, some by molecular chaperones, some by both approaches and some are resistant to these strategies (Anderson et al., 2006; Smith et al., 2013; Anderson et al., 2014). It is therefore highly likely that Piezo1 mutants may also display this behaviour.

In summary, here we showed that two loss-of-function PIEZO1 variants linked to different diseases show reduced stability at the protein level which correlates with increased ubiquitination. For both mutants, proteasome inhibition increased the protein amount implicating proteasomal degradation in their turnover. For one of these mutants (G2029R) we could boost the membrane targeting and stretchactivated currents via proteasomal inhibition. This suggests that in the future utilizing proteasomal inhibitors in use in the clinic in combination with a precision medicine-based approach we may be able to rectify the mis-localization and reduced function of Piezol channels associated with a subset of Piezo1-channel mediated channelopathies.

\section{DATA AVAILABILITY STATEMENT}

The raw data supporting the conclusion of this article will be made available by the authors, without undue reservation.

\section{AUTHOR CONTRIBUTIONS}

ZZ did western blot, confocal imaging and live cell labeling experiments. JL did patch clamp experiments. All authors conceived of the study and wrote the manuscript.

\section{FUNDING}

CC is supported by an NSW Health EMCR Fellowship. The experiments were in part supported by the Victor Chang Cardiac Research Institute Innovation Centre, funded by the NSW Government. BM is supported by a National Health and Medical Research Council of Australia Principal Research Fellowship (APP1135974). 


\section{REFERENCES}

Albarrán-Juárez, J., Iring, A., Wang, S., Joseph, S., Grimm, M., Strilic, B., et al. (2018). Piezo1 and Gq/G11 Promote Endothelial Inflammation Depending on Flow Pattern and Integrin Activation. J. Exp. Med. 215, 2655-2672. doi:10.1084/ jem. 20180483

Albuisson, J., Murthy, S. E., Bandell, M., Coste, B., Louis-Dit-Picard, H., Mathur, J., et al. (2013). Dehydrated Hereditary Stomatocytosis Linked to Gain-OfFunction Mutations in Mechanically Activated PIEZO1 Ion Channels. Nat. Commun. 4, 1884. doi:10.1038/ncomms2899

Anderson, C. L., Delisle, B. P., Anson, B. D., Kilby, J. A., Will, M. L., Tester, D. J., et al. (2006). Most LQT2 Mutations Reduce Kv11.1 (hERG) Current by a Class 2 (Trafficking-deficient) Mechanism. Circulation 113, 365-373. doi:10.1161/ CIRCULATIONAHA.105.570200

Anderson, C. L., Kuzmicki, C. E., Childs, R. R., Hintz, C. J., Delisle, B. P., and January, C. T. (2014). Large-scale Mutational Analysis of Kv11.1 Reveals Molecular Insights into Type 2 Long QT Syndrome. Nat. Commun. 5, 5535. doi:10.1038/ncomms6535

Arlow, T., Scott, K., Wagenseller, A., and Gammie, A. (2013). Proteasome Inhibition Rescues Clinically Significant Unstable Variants of the Mismatch Repair Protein Msh2. Proc. Natl. Acad. Sci. U S A. 110, 246-251. doi:10.1073/ pnas. 1215510110

Bae, C., Gnanasambandam, R., Nicolai, C., Sachs, F., and Gottlieb, P. A. (2013). Xerocytosis Is Caused by Mutations that Alter the Kinetics of the Mechanosensitive Channel PIEZO1. Proc. Natl. Acad. Sci. U S A. 110, E1162-E1168. doi:10.1073/pnas.1219777110

Buyan, A., Cox, C. D., Barnoud, J., Li, J., Chan, H. S. M., Martinac, B., et al. (2020). Piezo1 Forms Specific, Functionally Important Interactions with Phosphoinositides and Cholesterol. Biophys. J. 119, 1683-1697. doi:10.1016/ j.bpj.2020.07.043

Cao, X., Zhou, Z., Tian, Y., Liu, Z., Cheng, K. O., Chen, X., et al. (2021). Opposing Roles of E3 Ligases TRIM23 and TRIM21 in Regulation of Ion Channel ANO1 Protein Levels. J. Biol. Chem. 296, 100738, 2021 . Jan-Jun. doi:10.1016/ j.jbc.2021.100738

Chen, D., Frezza, M., Schmitt, S., Kanwar, J., and Dou, Q. P. (2011). Bortezomib as the First Proteasome Inhibitor Anticancer Drug: Current Status and Future Perspectives. Curr. Cancer Drug Targets 11, 239-253. doi:10.2174/ 156800911794519752

Christianson, J. C., and Ye, Y. (2014). Cleaning up in the Endoplasmic Reticulum: Ubiquitin in Charge. Nat. Struct. Mol. Biol. 21, 325-335. doi:10.1038/ nsmb. 2793

Coste, B., Mathur, J., Schmidt, M., Earley, T. J., Ranade, S., Petrus, M. J., et al. (2010). Piezo1 and Piezo2 Are Essential Components of Distinct Mechanically Activated Cation Channels. Science 330, 55-60. doi:10.1126/science.1193270

Coste, B., Murthy, S. E., Mathur, J., Schmidt, M., Mechioukhi, Y., Delmas, P., et al. (2015). Piezo1 Ion Channel Pore Properties Are Dictated by C-Terminal Region. Nat. Commun. 6, 7223. doi:10.1038/ncomms8223

Cox, C. D., Bae, C., Ziegler, L., Hartley, S., Nikolova-Krstevski, V., Rohde, P. R., et al. (2016). Removal of the Mechanoprotective Influence of the Cytoskeleton Reveals PIEZO1 Is Gated by Bilayer Tension. Nat. Commun. 7, 10366. doi:10.1038/ncomms10366

Cox, C. D., Bavi, N., and Martinac, B. (2019). Biophysical Principles of IonChannel-Mediated Mechanosensory Transduction. Cell Rep 29, 1-12. doi:10.1016/j.celrep.2019.08.075

Douguet, D., Patel, A., Xu, A., Vanhoutte, P. M., and Honoré, E. (2019). Piezo Ion Channels in Cardiovascular Mechanobiology. Trends Pharmacol. Sci. 40, 956-970. doi:10.1016/j.tips.2019.10.002

Duchemin, A. L., Vignes, H., and Vermot, J. (2019). Mechanically Activated Piezo Channels Modulate Outflow Tract Valve Development through the Yap1 and Klf2-Notch Signaling axis. eLife 8. doi:10.7554/eLife.44706

Faucherre, A., Moha Ou Maati, H., Nasr, N., Pinard, A., Theron, A., Odelin, G., et al. (2020). Piezo1 Is Required for Outflow Tract and Aortic Valve Development. J. Mol. Cel Cardiol 143, 51-62. doi:10.1016/j.yjmcc.2020.03.013

Fotiou, E., Martin-Almedina, S., Simpson, M. A., Lin, S., Gordon, K., Brice, G., et al. (2015). Novel Mutations in PIEZO1 Cause an Autosomal Recessive Generalized Lymphatic Dysplasia with Non-immune Hydrops Fetalis. Nat. Commun. 6, 8085. doi:10.1038/ncomms9085
Glogowska, E., Schneider, E. R., Maksimova, Y., Schulz, V. P., Lezon-Geyda, K., $\mathrm{Wu}$, J., et al. (2017). Novel Mechanisms of PIEZO1 Dysfunction in Hereditary Xerocytosis. Blood 130, 1845-1856. doi:10.1182/blood-2017-05-786004

Jiang, F., Yin, K., Wu, K., Zhang, M., Wang, S., Cheng, H., et al. (2021). The Mechanosensitive Piezol Channel Mediates Heart Mechano-Chemo Transduction. Nat. Commun. 12, 869. doi:10.1038/s41467-021-21178-4.(2021

Kanner, S. A., Shuja, Z., Choudhury, P., Jain, A., and Colecraft, H. M. (2020). Targeted Deubiquitination Rescues Distinct Trafficking-Deficient Ion Channelopathies. Nat. Methods 17, 1245-1253. doi:10.1038/s41592-020-00992-6

Kao, S. H., Wang, W. L., Chen, C. Y., Chang, Y. L., Wu, Y. Y., Wang, Y. T., et al. (2015). Analysis of Protein Stability by the Cycloheximide Chase Assay. Bio Protoc. 5. doi:10.21769/BioProtoc.1374

Knoepp, F., Ashley, Z., Barth, D., Baldin, J. P., Jennings, M., Kazantseva, M., et al. (2020). Shear Force Sensing of Epithelial Na+ Channel (ENaC) Relies on $\mathrm{N}$-Glycosylated Asparagines in the palm and Knuckle Domains of aENaC. Proc. Natl. Acad. Sci. U S A. 117, 717-726. doi:10.1073/pnas.1911243117

Lai, A., Chen, Y. C., Cox, C. D., Jaworowski, A., Peter, K., and Baratchi, S. (2021). Analyzing the Shear-Induced Sensitization of Mechanosensitive Ion Channel Piezo-1 in Human Aortic Endothelial Cells. J. Cel Physiol 236, 2976-2987. doi:10.1002/jcp.30056

Lewis, A. H., and Grandl, J. (2015). Mechanical Sensitivity of Piezo1 Ion Channels Can Be Tuned by Cellular Membrane Tension. eLife 4. doi:10.7554/eLife.12088

Li, J., Hou, B., Tumova, S., Muraki, K., Bruns, A., Ludlow, M. J., et al. (2014). Piezo1 Integration of Vascular Architecture with Physiological Force. Nature 515, 279-282. doi:10.1038/nature13701

Li, J. V., Ng, C.-A., Cheng, D., Yao, M., Guo, Y., Yu, Z.-Y., et al. (2021). Modified N-Linked Glycosylation Status Predicts Trafficking Defective Human Piezo1 Channel Mutations. Commun. Biol. 4, 1038

Lukacs, V., Mathur, J., Mao, R., Bayrak-Toydemir, P., Procter, M., Cahalan, S. M., et al. (2015). Impaired PIEZO1 Function in Patients with a Novel Autosomal Recessive Congenital Lymphatic Dysplasia. Nat. Commun. 6, 8329. doi:10.1038/ncomms9329

Maneshi, M. M., Ziegler, L., Sachs, F., Hua, S. Z., and Gottlieb, P. A. (2018). Enantiomeric A $\beta$ Peptides Inhibit the Fluid Shear Stress Response of PIEZO1. Sci. Rep. 8, 14267. doi:10.1038/s41598-018-32572-2

Martinac, B., and Cox, C. D. (2017). Comprehensive Biophysics, 7. Elsevier.

Mohanraj, K., Wasilewski, M., Benincá, C., Cysewski, D., Poznanski, J., Sakowska, P., et al. (2019). Inhibition of Proteasome Rescues a Pathogenic Variant of Respiratory Chain Assembly Factor COA7. EMBO Mol. Med. 11. doi:10.15252/ emmm.201809561

Murthy, S. E., Dubin, A. E., and Patapoutian, A. (2017). Piezos Thrive under Pressure: Mechanically Activated Ion Channels in Health and Disease. Nat. Rev. Mol. Cel Biol 18, 771-783. doi:10.1038/nrm.2017.92

Nonomura, K., Lukacs, V., Sweet, D. T., Goddard, L. M., Kanie, A., Whitwam, T., et al. (2018). Mechanically Activated Ion Channel PIEZO1 Is Required for Lymphatic Valve Formation. Proc. Natl. Acad. Sci. U S A. 115, 12817-12822. doi:10.1073/pnas.1817070115

Poole, K. (2021). The Diverse Physiological Functions of Mechanically Activated Ion Channels in Mammals. Annu. Rev. Physiol. 84. doi:10.1146/annurevphysiol-060721-100935

Ranade, S. S., Qiu, Z., Woo, S. H., Hur, S. S., Murthy, S. E., Cahalan, S. M., et al. (2014). Piezo1, a Mechanically Activated Ion Channel, Is Required for Vascular Development in Mice. Proc. Natl. Acad. Sci. U S A. 111, 10347-10352. doi:10.1073/pnas.1409233111

Ridone, P., Pandzic, E., Vassalli, M., Cox, C. D., Macmillan, A., Gottlieb, P. A., et al. (2020). Disruption of Membrane Cholesterol Organization Impairs the Activity of PIEZO1 Channel Clusters. J. Gen. Physiol. 152, e201912515. In press. doi:10.1085/jgp.201912515

Robertson, G. A., and January, C. T. (2006). HERG Trafficking and Pharmacological rescue of LQTS-2 Mutant Channels. Handb Exp. Pharmacol. 2006, 349-355. doi:10.1007/3-540-29715-4_14

Rode, B., Shi, J., Endesh, N., Drinkhill, M. J., Webster, P. J., Lotteau, S. J., et al. (2017). Piezol Channels Sense Whole Body Physical Activity to Reset Cardiovascular Homeostasis and Enhance Performance. Nat. Commun. 8 350. doi:10.1038/s41467-017-00429-3

Ron, D., and Walter, P. (2007). Signal Integration in the Endoplasmic Reticulum Unfolded Protein Response. Nat. Rev. Mol. Cel Biol 8, 519-529. doi:10.1038/ nrm2199 
Rusconi, R., Scalmani, P., Cassulini, R. R., Giunti, G., Gambardella, A., Franceschetti, S., et al. (2007). Modulatory Proteins Can rescue a Trafficking Defective Epileptogenic Nav1.1 Na+ Channel Mutant. J. Neurosci. 27, 11037-11046. doi:10.1523/jneurosci.3515-07.2007

Saotome, K., Murthy, S. E., Kefauver, J. M., Whitwam, T., Patapoutian, A., and Ward, A. B. (2018). Structure of the Mechanically Activated Ion Channel Piezo1. Nature 554, 481-486. doi:10.1038/nature25453

Smith, J. L., Reloj, A. R., Nataraj, P. S., Bartos, D. C., Schroder, E. A., Moss, A. J., et al. (2013). Pharmacological Correction of Long QT-Linked Mutations in KCNH2 (hERG) Increases the Trafficking of Kv11.1 Channels Stored in the Transitional Endoplasmic Reticulum. Am. J. Physiol. Cel Physiol 305, C919-C930. doi:10.1152/ajpcell.00406.2012

Syeda, R., Florendo, M. N., Cox, C. D., Kefauver, J. M., Santos, J. S., Martinac, B., et al. (2016). Piezo1 Channels Are Inherently Mechanosensitive. Cel Rep 17, 1739-1746. doi:10.1016/j.celrep.2016.10.033

Syeda, R. (2021). Physiology and Pathophysiology of Mechanically Activated PIEZO Channels. Annu. Rev. Neurosci. 44, 383-402. doi:10.1146/annurevneuro-093020-120939

Tannous, A., Pisoni, G. B., Hebert, D. N., and Molinari, M. (2015). N-linked SugarRegulated Protein Folding and Quality Control in the ER. Semin. Cel Dev Biol 41, 79-89. doi:10.1016/j.semcdb.2014.12.001

Vandenberg, J. I., Perry, M. D., Perrin, M. J., Mann, S. A., Ke, Y., and Hill, A. P. (2012). hERG $\mathrm{K}(+)$ Channels: Structure, Function, and Clinical Significance. Physiol. Rev. 92, 1393-1478. doi:10.1152/ physrev.00036.2011

Vero Li, J., D Cox, C., and Martinac, B. (2021). The Anchor Domain Is Critical for Piezo1 Channel Mechanosensitivity. Channels (Austin) 15, 438-446. doi:10.1080/19336950.2021.1923199

Wang, Y., Shen, T., Fang, P., Zhou, J., Lou, K., Cen, Z., et al. (2018). The Role and Mechanism of Chaperones Calnexin/Calreticulin in Which ALLN Selectively
Rescues the Trafficking Defective of HERG-A561v Mutation. Biosci. Rep. 38. doi:10.1042/BSR20171269

Yu, Z.-Y., Gong, H., Kesteven, S., Guo, Y., Wu, J., Li, J., et al. (2021). Piezol Is the Cardiac Mechanosensor that Initiates the Hypertrophic Response to Pressure Overload. Researchsquare. doi:10.21203/rs.3.rs-895561/v1

Zarychanski, R., Schulz, V. P., Houston, B. L., Maksimova, Y., Houston, D. S. Smith, B., et al. (2012). Mutations in the Mechanotransduction Protein PIEZO1 Are Associated with Hereditary Xerocytosis. Blood 120, 1908-1915. doi:10.1182/blood-2012-04-422253

Zhang, T., Chi, S., Jiang, F., Zhao, Q., and Xiao, B. (2017). A Protein Interaction Mechanism for Suppressing the Mechanosensitive Piezo Channels. Nat. Commun. 8, 1797. doi:10.1038/s41467-017-01712-z

Conflict of Interest: The authors declare that the research was conducted in the absence of any commercial or financial relationships that could be construed as a potential conflict of interest.

Publisher's Note: All claims expressed in this article are solely those of the authors and do not necessarily represent those of their affiliated organizations, or those of the publisher, the editors and the reviewers. Any product that may be evaluated in this article, or claim that may be made by its manufacturer, is not guaranteed or endorsed by the publisher.

Copyright (®) 2021 Zhou, Li, Martinac and Cox. This is an open-access article distributed under the terms of the Creative Commons Attribution License (CC BY). The use, distribution or reproduction in other forums is permitted, provided the original author(s) and the copyright owner(s) are credited and that the original publication in this journal is cited, in accordance with accepted academic practice. No use, distribution or reproduction is permitted which does not comply with these terms. 